\title{
On some conjectures concerning pseudo-convex domains.
}

\author{
By Sin Hitotumatu
}

(Received Dec. 8, 1953)

The famous problem of E. E. Levi [24], asking whether every pseudo-convex domain is a domain of regularity or not, still awaits a complete solution, though there are a number of deep results concerning this problem. ${ }^{1)}$ For this, the theory of pluri-subharmonic functions due to P. Lelong ([20]-[23]), K. Oka ([26]), H. J. Bremermann ([10]) and G. O. Thorin ([35]) seems to give a powerful method. This theory allows us in particular to restate the problem of Levi in several forms, in which it may probably be easier to resolve.

In the first and the second chapters of the present paper, we shall give, for later use, a brief sketch of definitions and the main results of the theory of pluri-subharmonic functions and of the domains convex with respect to these functions, respectively. Then, we shall show in the third chapter, that there exist some intimate relations among the conjectures concerning the pseudo-convex domains and the pluri-subharmonic functions, as considered by several authors. ${ }^{2)}$

For the sake of simplicity, we treat only the domains univalent and finite (not necessarily bounded) in the space $\mathbb{5}^{n}$ of $n$ complex variables $\left(z_{1}, \cdots, z_{n}\right)$. A point or a vector $\left(z_{1}, \cdots, z_{n}\right)$ in our space $\mathbb{5}^{n}$ is sometimes denoted merely by $\left(z_{j}\right)$ or by $z$.

1) After completion of this manuscript, the author has been told that Prof. K. Oka solved the problem of Levi affirmatively in its original form. His results will soon appear in his paper [28]. However, it may not be meaningless to consider the relations of this problem to other famous conjectures, and after Oka's results, we obtain some results, seems to be new, as $4 \mathrm{~d}$ and $4 \mathrm{e}$ in Chapter III.

2) For example, Behnke-Stein [5], H. Cartan [13], [14], and Thullen [38], 


\section{Pluri-subharmonic functions.}

\section{$\S 1$. Definitions of pluri-subharmonic functions.}

DEFINITION 1. A real-valued function $V(z)$ in a domain $D$ of our

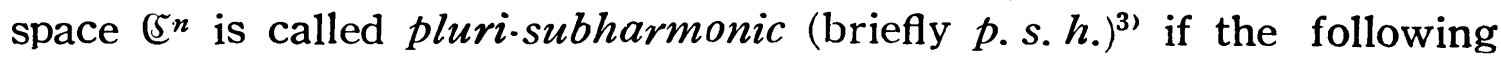
three conditions (P1)-(P3) hold:

(P1) $-\infty \leqq V<\infty$, and $V \neq-\infty$.

(P2) $V$ is upper semi-continuous in $D$.

(P3) On any analytic plane of real dimension $2, z_{j}=z_{j}^{0}+a_{j} t$, $(j=1, \cdots, n)$ where $t$ is a complex parameter and $\left(a_{j}\right) \neq(0)$, the function $V\left(z^{0}+\mathrm{a} \cdot t\right)$ is a subharmonic function of $t$ or the constant $-\infty$.

The condition (P3) is clearly equivalent to the following (P4) and (P5) :

(P4) If we fix for any $j$, the $(n-1)$ variables $z_{k}=z_{k}^{0}(k \neq j)$, the function

$$
V\left(z_{1}^{0}, \cdots, z_{j-1}^{0}, z_{j}, z_{j+1}^{0}, \cdots, z_{n}^{0}\right)
$$

is a subharmonic function of $z_{j}$ or the constant $-\infty$.

(P5) After changing the coordinates by a unitary transformation, the same properties still hold.

P. Lelong ([20]) proved that under the conditions (P1) and (P4), the condition (P2) may be replaced by a weaker condition:

(P2') $\quad V$ is bounded from above in any compact subset of $D$.

But the proof being somewhat complicated, we adopt $a$ priori the condition (P2) in the definition

If a polycylinder $S:\left|z_{j}-z_{j}^{0}\right| \leqq r_{j}$ is contained in $D$, we can integrate $V$ on $S$ or on its edge $C:\left|z_{j}-z_{j}^{0}\right|=r_{j}$, provided that we admit the value $-\infty$ for the integral, and from (P4) we have

$$
\begin{aligned}
& \left(\mathrm{P} 4^{\prime}\right) \quad V\left(z^{0}\right) \leqq L\left(V, z^{0}, r\right) \equiv \begin{array}{c}
1 \\
(2 \pi)^{n}
\end{array} \int_{0}^{2 \pi} \cdots \int_{0}^{2 \pi} V\left(z_{j}^{0}+r_{j} e^{i \theta}\right) d \theta_{1} \cdots d \theta_{n}, \\
& \left(\mathrm{P}^{\prime \prime}\right) \quad V\left(z^{0}\right) \leqq A\left(V, z^{0}, r\right) \equiv \frac{1}{\pi^{n} r_{1}^{2} \cdots r_{n}^{2}} \int \cdots \int_{S} V\left(z_{j}\right) d \omega,
\end{aligned}
$$

where $d \omega$ is the volume element of $S$. Conversely, under (P1) and

3) This name is due to Lelong. Oka and Bremermann called it "pseudo-convex function". 
(P2), the condition (P4) may be replaced by $\left(\mathrm{P}^{\prime}\right)$ or $\left(\mathrm{P} 4^{\prime \prime}\right)$. Also we have the following, integrating $\left(\mathrm{P} 4^{\prime}\right)$ over $\sum r_{j}^{2}=r^{2}$ :

Proposition 1. A p.s. h. function is subharmonic with respect to $2 n$ real variables $x_{1}, \cdots, x_{n}, y_{1}, \cdots, y_{n}$ where we put $z_{j}=x_{j}+i y_{j}$.

Corollary. For a p.s.h. function $V(z)$, the set $\{z \mid V(z)=-\infty\}$ is of outer capacity 0 for the Newton potential in the $2 n$-dimensional real space. (H. Cartan [12]).

Proposition 2. If $f(z) \neq 0$ is holomorphic and $|f(z)|$ is one-valued in $D, \log |f(z)|$ is p.s.h. in $D$. A positive constant multiple of p. s. h., the sum and the maximum of a finite number of p.s.h. functions are also p.s.h. The limit of a decreasing sequence of p.s.h. function is p. s. h. or the constant $-\infty$.

PRoposition 3. If a family of p.s. h. functions $\left\{V_{\nu}\right\}_{\nu}$ is uniformly bounded from above in every compact subset of $D$, and $V \equiv \sup _{\nu} V_{\nu}$ is continuous in $D$, then $V$ itself is also p.s. h. in $D$.

Proposition 4. Let $p$ be a preassigned positive integer or $+\infty$. For any compact subset of $D$, a p. s. h. function $V(z)$ is the limit of a decreasing sequence of p.s. h. functions continuously $p$-times differentiable (i. e., of the differentiable class $\mathrm{C}^{p}$ ).

In fact, when $p<\infty$, this is proved similarly as the case of sub. harmonic functions, repeating the process of integral mean ( $T$. Radô [30], p. 11). If $p=\infty$, this can be provee by the method of regularisation by functions converging to "Dirac's $\delta$ ". (See Lelong [23], pp. 183-184).

Definition 2. Let $D \subset B$ and let $U$ and $V$ be p.s.h. in $D$ and $B$ respectively. If $U(z) \equiv V(z)$ in $D, V$ is called a p.s.h.-continuation of $U$ into $B$. Of course, the p.s.h. continuation is not unique in general.

\section{$\$ 2$. Condition of Levi-Krzoska.}

When the function $V$ has continuous derivatives up to the second order, the condition (P3) is equivalent to the following:

$\left(\mathrm{P} 3^{\prime}\right)$ The Hermitian form $\sum_{j, k=1}^{n} \frac{\partial^{2} V}{\partial z_{j} \partial \bar{z}_{k}} \xi_{j} \bar{\xi}_{k}$ is positive semi-definite.

However, when the function $V$ is not differentiable in the ordinary sense, we can replace the derivatives in the sense of the "distribution" 
of L. Schwartz ([34]) and (P3) is always equivalent to the condition: $\left(\mathrm{P}^{\prime \prime}\right)$ For every pair of complex constants $\left(\xi_{1}, \cdots, \xi_{n}\right)$, the Hermitian from $\sum_{j, k=1}^{n} \frac{\partial^{2} V}{\partial z_{j} \partial \bar{z}_{k}} \xi_{j} \bar{\xi}_{k}$ given by the derivatives as: distributions, is always a non-negative measure.

Definition 3. We shall say that a p.s.h. function $V$ is strictly pluri-subharmonic (briefly s.p.s.h.) if the Hermitian form given in $\left(\mathrm{P} 3^{\prime \prime}\right)$ is a strictly positive measure unless $\left(\xi_{j}\right)=(0)$. When $V$ is of class $\mathrm{C}^{2}$, this condition is equivalent to the fact the Hermitian form in $\left(\mathrm{P}^{\prime}\right)$ is strictly positive.definite.

Adding $\varepsilon \sum_{j=1}^{n}\left|z_{j}\right|^{2}$ to $V(z)$ and letting $\varepsilon \rightarrow+0$, we have, from Proposition 4 ,

Proposition 4 bis. In any compact subset of $D$, a p.s. h. function $V$ is the limit of a decreasing sequence of s.p.s.h. functions of class $\mathrm{C}^{\infty}$.

Proposition 5. By an analytic transformation, a p.s.h. function is mapped into a p.s.h. function. Also on a complex-analytic variety $z_{j}=\phi_{j}\left(t_{1}, \cdots, t_{q}\right)(j=1, \cdots, n)$ the function $V(\varphi(\mathrm{t}))$ is $\mathrm{p} . \mathrm{s} . \mathrm{h}$. with respect to the variables $t_{1}, \cdots, t_{q}$ or the constant $-\infty$.

Definition 4. A real-valued function $\Phi(z)$ of class $\mathrm{C}^{3}$ is said to satisfy the condition of Levi-Krzoska at a point $z^{0}$ if for any pair of complex numbers $\xi_{1}, \cdots, \xi_{n}$ of which at least one is not zero, satisfying

$$
\sum_{j=1}^{n}\left(\frac{\partial \Phi}{\partial z_{j}}\right)_{\left(z^{0}\right)} \xi_{j}=0
$$

we have

$$
\sum_{j, k=1}^{n}\left(\frac{\partial^{2} \Phi}{\partial z_{j} \partial \bar{z}_{k}}\right)_{\left(z^{0},\right.} \xi_{j}, \bar{\xi}_{k}>0 .{ }^{4)}
$$

In case of $n=2$, this condition is equivalent to the condition of Levi ([24]) :

$$
\mathcal{L}(\Phi) \equiv-\left|\begin{array}{ccc}
0 & \partial \Phi / \partial z_{1} & \partial \Phi / \partial z_{2} \\
\partial \Phi / \partial \bar{z}_{1} & \partial^{2} \Phi / \partial z_{1} \partial \bar{z}_{1} & \partial^{2} \Phi / \partial z_{2} \partial \bar{z}_{1} \\
\partial \Phi / \partial \bar{z}_{2} & \partial^{2} \Phi / \partial z_{1} \partial \bar{z}_{2} & \partial^{2} \Phi / \partial z_{2} \partial \bar{z}_{2}
\end{array}\right|>0 .
$$

4) J. Krzoska [18]. See also S. Bochner [7], pp. 228-231. For the condition of Levi, see also Behnke-Thullen [6], p. 29. 
Proposition 6. A function $\Phi(z)$ of class $\mathrm{C}^{3}$ is written into the from $\Phi=U \cdot V$ in a neighborhood $N$ of $z^{0}$, where $U$ and $V$ satisfy the conditions that they are both of class $\mathrm{C}^{3}, V$ is p.s.h., $0<U(<\infty)$ in $N$ and $V\left(z^{0}\right)=0$, if and only if $\Phi\left(z^{0}\right)=0$ and $\Phi$ satisfies the condition of Levi-Krzoska at $z^{0}$.

CoROllary. If a function $\Phi(z)$ satisfies the condition of LeviKrzoska at $z^{0}$, there exists a complex-analytic variety of $(n-1)$-dimension $\mathfrak{B}$, which we may select as a quadratic veriety, passing the point $z^{0}$ such that in a neighborhood of $z^{0}, \mathfrak{S}$ lies in the part $\left\{\Phi>\Phi\left(z^{0}\right)\right\}$ except the point $z^{0}$.

\section{§3. Hartogs functions:}

According to M. Brelot [9], a function $V(z)$ in a plane region is said to be quasi-subharmonic if there exists a subharmonic function $U(z)$ such that $V \leqq U$ and equality holds except on a set of logarithmic outer capacity 0 .

Definition 5. A function $V(z)$ in a domain $D$ of our space $\sigma^{n}$ is called quasi-pluri-subharmonic (briefly q.p.s.h.) if it satisfies the conditions ( $\mathrm{P} 1)$, $\left(\mathrm{P}^{\prime}\right)$ in $\$ 1$ and ( $\mathrm{P} 3$ bis) obtained by replacing the word "subharmonic" by "quasi-subharmonic" in (P3).

Proposition 2 bis. A p.s.h. function is evidently q. p.s. h. The properties obtained by replacing the word "p.s.h." in Proposition 2 by "q. p. s. h." still hold. Also the supremum of a family of q. p. s. h. functions uniformly bounded from above in any compact subset of $D$ is also q. p. s. h. in $D$.

Proposition 7. If $V$ is q. p.s. h. in $D$, the function

is p. s. h. in $D^{6}{ }^{6}$

$$
V^{*}(z) \equiv \limsup _{\zeta \rightarrow z} V(\zeta)
$$

CoRollary. (Generalization of Proposition 3). If a family of p. s. h. functions $\left\{V_{\nu}\right\}_{\nu}$ is uniformly bounded from above in any compact subset of $D$, the function

$$
V \equiv \sup _{\nu} V_{\nu} \equiv\left(\sup _{\nu} V_{\nu}\right)^{*}
$$

5) Lelong [23], p. 190. For $n=2$, Oka [26], p. 48.

6) Lelong [20], p. 334. Another proof is given in Bremermann [10], p. 29, but the latter proof seems to be somewhat incomplete, because it is not evident a priori that "the set $\left\{z \mid V(z)=V^{*}(z)\right\}$ lies dense in $D^{\prime}$. 
is p.s. h. in $D$. Especially if $\{\nu\}=\{1,2, \cdots\}$, we have

$$
V \equiv \lim _{\nu \rightarrow \infty} \sup ^{*} V_{\nu} \equiv\left(\lim _{\nu \rightarrow \infty} \sup _{\nu} V^{*}=\lim _{l \rightarrow \infty}\left(\sup _{\nu \geq l} V_{\nu}\right)\right.
$$

and the function $V$ is p.s.h., unless $V \equiv-\infty$.

Now, following S. Bochner and W. T. Martin ([8], Chap. VII), we introduce the notion of Hartogs functions as follows ${ }^{7)}$ :

Definition 6. Take a family of functions $\mathfrak{r}$ in a domain $D$, and consider the following conditions:

(H0) If a function $f(z) \neq 0$ is one-valued and holomorphic in $D$, then $\log |f(z)| \in \mathfrak{F}$.

(H1) If $\phi, \psi \in \mathfrak{F}$ and $c$ is a non-negative constant, we have $\phi+\psi \in \mathfrak{F}$ and $c \phi \in \mathfrak{r}$.

(H2) If $\left\{\phi_{m}\right\}_{m} \in \mathfrak{F}$ is a decreasing sequence, we have, $\phi \equiv \lim _{m \rightarrow \infty} \phi_{m} \in \mathfrak{F}$, unless $\phi \equiv-\infty$.

(H3) If $\left\{\boldsymbol{\phi}_{\nu}\right\}_{\nu}$ is a subfamily of $\mathfrak{F}$ uniformly bounded from above in any compact subset of $D$, we have $\sup _{\nu} \phi_{\nu} \equiv \phi \in \Re$.

(H4) $\phi \in \mathfrak{F}$ implies $\phi^{*} \in \mathfrak{F}$.

(H5) If $\left\{\boldsymbol{\phi}_{\nu}\right\}_{\nu}$ is a subfamily of $\widetilde{F}$ uniformly bounded from above in any compact subset of $D$, we have $\sup _{\nu}^{*} \varphi_{\nu} \equiv \phi \in \mathfrak{T}$.

Then, a function belonging to any family $\mathfrak{s}$ satisfying the conditions $(\mathrm{H} 0),(\mathrm{H} 1),(\mathrm{H} 2),(\mathrm{H} 3)$ and $(\mathrm{H} 4)$ or $(\mathrm{H} 0),(\mathrm{H} 1),(\mathrm{H} 2)$ and $(\mathrm{H} 5)$ is called a Hartogs function or a modified Hartogs function, respectively. Evidently the former notion is wider than the latter.

The above propositions show that $a$ Hartogs function or a modified Hartogs function is q. p.s. h. or p. s. h., respectively. Are the converses of these facts true? This seems to be one of the most important problems in the theory of pluri-subharmonic functions; we shall discuss it in III.

The application of the Riesz decomposition (Lelong [21]) of a p. s. h. function as an ordinary subharmonic function (cf. Proposition 1) seems to be so limited for our present theory that we shall omit here.

7) It seems to me thrat in the recent papers, they are using the word "Hartogs functions" as the synonym of the "pluri-subharmonic functions", but here, we must distinguish these two terminologies. 


\section{Domains convex with respect to p.s.h. functions.}

\section{$\S 4$. P-convex domains.}

Hereafter the notation $A C(B$ mean that $\bar{A}$, the closure of $A$, is contained in the open kernel of $B$. We shall say that a domain $D$ is the limit of an exhaustion of domains $D_{m}$ if $D_{m}$ are all bounded, $D_{m}\left(\left(D_{m+1}\left(C D\right.\right.\right.$ and $\underset{m}{\cup} D_{m}=D$.

As an analogy of the regular-convexity, ${ }^{8)}$ we shall introduce the following notion due to Lelong [23]:

Definition 7. A domain $D$ in our space $\mathbb{C}^{n}$ is called convex with respect to pluri-subharmonic functions (briefly P-convex), if for every compact subset $\Delta$ of $D$ there exist two sets $\Delta^{\prime}$ and $G$ such that $\Delta \subset \Delta^{\prime}$ ( $\left(D\right.$ and $G$ is dense in $D-\Delta^{\prime}$, and that for every point $p$ of $G$ there exists a function $V_{p}$ p.s.h. in $D$ satisfying $V_{p}(p)>\sup _{z \in \Delta} V_{p}(z)$. We shall denote by $\left(\mathrm{C}_{0}\right)$ the family of all P-convex domains.

From the regular-convexity of a domain of regularity, ${ }^{8)}$ it is evident that every domain of regularity is always P-convex. The converse of this fact is a problem which we shall discuss in III. Also, the image of a P-convex domain by an analytic transformation and the intersection of a finite number of $\mathrm{P}$-convex domains are again P-convex.

Definition 8. A domain $D$ is locally P-convex or Cartan pseudoconvex ${ }^{9)}$ if for every boundary-point $p$ of $D$, there exists a hypersphere $B$ whose center is at $p$, such that $D \cap B$ is P-convex or a domain of regularity, respectively. We denote by $\left(\mathrm{C}_{0}^{l}\right)$ the family of all locally P-convex domains. It is evident that a Cartan pseudo-convex domain is locally P-convex.

Definition 9. A domain $D$ is said to belong to the class $\left(\Gamma_{0}\right)$ if there exists a function $V$ p.s.h. in $D$ such that, for every constant $\alpha$, the set $\{V<\alpha\}$ is bounded and ( $D$.

DEFINITION 10. Denote by $d(z)$ or $d^{\prime}(z)$ the minimal distance from a point $z$ to the boundary of a domain $D$, taking the distance of two points $\left(\zeta^{(1)}\right)$ and $\left(\zeta^{(2)}\right)$ as $\sqrt{\sum_{j=1}^{n}\left|\zeta_{j}^{(1)}-\zeta_{j}^{(2)}\right|^{2}}$ or $\max _{1 \leq j \leq n}\left|\zeta_{j}^{(1)}-\zeta_{j}^{(2)}\right|$, respective-

8) Cartan-Thullen [15]. See also, H. Cartan [11] and Behnke-Stein [4].

9) The latter may be called "domain of locally-regularity", but instead of this, the terminology "Cartan pseudo-convex" is ordinarily used. 
1y. Also for a unit vector $\mathrm{a}=\left(a_{1}, \cdots, a_{n}\right)$ (i. e., $\sum_{j=1}^{n}\left|a_{j}\right|^{2}=1$ ), the minimal distance from $z$ to the boundary of $D$ along an analytic plane parallel to $z_{j}=a_{j} \cdot t$, where $t$ is a complex parameter, is denoted by $d_{\mathrm{a}}(z)$. A domain $D$ is said to belong to the class $\left(I^{\prime}\right),\left(I^{\prime}\right)$ or $\left(I_{1}\right)$ if the function $-\log d(z),-\log d^{\prime}(z)$ or $-\log d_{\mathrm{a}}(z)$ for every unit-vector a are p.s.h. in $D$, respectively. Also we write $D \in\left(\Gamma_{*}\right)$ or $D \in\left(\Gamma_{*}^{\prime}\right)$ if $-\log d(z)$ or $-\log d^{\prime}(z)$ is p.s. h. in a boundary-strip $\{z \mid d(z)<\varepsilon\}$ of $D$, respectively.

Proposition 8. We have the relations of inclusion as follows:

$$
\begin{gathered}
\left(I_{1}\right) \subset\left(I^{\prime}\right) \subset\left(I_{*}^{\prime}\right)=\left(I^{\prime \prime}\right) \subset\left(I^{\prime \prime}\right) \subset\left(\mathrm{C}_{0}\right) \subset\left(\mathrm{C}_{0}^{t}\right) . \\
\left.I^{\prime \prime}\right)
\end{gathered}
$$

Proof. $\left(I_{1}^{\prime}\right) \subset\left(I^{\prime}\right),\left(I^{\prime \prime}\right)$ follows from Proposition 3 , for $d(z)=\inf _{\mathbf{a}} d_{\mathbf{a}}(z)$ and $d^{\prime}(z)=\inf c_{\mathrm{a}} \cdot d_{\mathrm{a}}(z),\left(c_{\mathrm{a}}>0\right) . \quad\left(I_{*}^{\prime}\right),\left(I_{*}^{\prime \prime}\right) \approx\left(I_{0}^{\prime}\right)$ is proved by putting $V(z)=\max \left[-\log \frac{\varepsilon}{2 \sqrt{n}},-\log d(z)\right.$ (or $\left.\left.d^{\prime}(z)\right), \sum_{j=1}^{n}\left|z_{j}\right|^{2}\right]^{10)}$ The remaining relations are obvious from their definitions.

CoROllary. When $n=1$, every domain belongs to $\left(I^{\prime}\right)$, and then is $\mathrm{P}$.convex.

Definition 11. A domain $D$ is said to belong to the class $\left(\mathrm{C}_{1}^{\alpha}\right)$, if it is a componsnt of the set $\{V<0\}$ where $V$ is a p.s.h. function in a domain $B$ such that $D((B$. Especially, when $V$ is s. p.s.h. and of class $\mathrm{C}^{\infty}$ in $B$, we write $D \in\left(\mathrm{C}_{1}^{\gamma}\right)$. A domain which is the limit of an exhaustion of domains of class $\left(\mathrm{C}_{1}^{\alpha}\right)$ or $\left(\mathrm{C}_{1}^{\gamma}\right)$ is denoted by $D \in\left(\mathrm{C}_{1}\right)$ or $D \in\left(\mathrm{C}_{1}^{*}\right)$, respectively.

By Proposition 4 bis, we have

Proposition 9. $\left(\mathrm{C}_{1}\right)=\left(\mathrm{C}_{1}^{*}\right)$ holds.

Also by a similar method as the approximation of a domain of regularity by polyhedral domains ${ }^{11}$, we have :

Proposition 10. $\left(\mathrm{C}_{0}\right) \subset\left(\mathrm{C}_{1}\right)$ holds.

\section{§. Oka pseudo-convex domains.}

Definition 12 . Let $n$ functions $\varphi_{j}(u, t)$ be continuous on $\Delta:|u|$ $\leqq 1,0 \leqq t \leqq 1$, holomorphic in $|u|<1$ when the parameter $t$ is fixed

10) When $D$ is bounded, that last term is unnecessary.

11) Weil [39]. See also Behnke-Stein [4], and Lelong [23]. 
in $[0,1]$, and assume that $\partial \varphi_{j} / \partial u$ are not all zero at any point on $\Delta$. Then the set $Q(t) \equiv\left\{z_{j}=\varphi_{j}(u, t)|| u \mid \leqq 1\right\}$ is called an analytic disc depending on a parameter $t$, and $B(t) \equiv\left\{z_{j}=\varphi_{j}(u, t)|| u \mid=1\right\}$ is called its shell.

Definition 13. A domain $D$ in our space (5n is called Oka pseudoconvex if the generalized continuity theorem (e. g., Behnke-Sommer [2]) in the form of an analytic disc holds on the boundary of $D$. More precisely, for every analytic disc depending on a parameter $t$ such that $\overline{Q(t)} \subset D(0<t)$ and $B(0)<D$, we have $Q(0) \subset D$. We denote by $\left(\mathrm{C}_{2}\right)$ the family of all the Oka pseudo-convex domains. (Oka [26]).

Since the set $\bigcup_{0 \leq t \leq 1}[B(t)] \cup[(Q(1)]$ is compact, we have easily that the limit of an exhaustion of Oka pseudo-convex domains is again Oka pseudo-convex. Now, by the maximum principle on subharmonic functions (e. g., Radó [30], p. 6), we have

PRoposition 11. $\left(\mathrm{C}_{1}^{\gamma}\right) \subset\left(\mathrm{C}_{2}\right)$ and then $\left(\mathrm{C}_{1}\right) \subset\left(\mathrm{C}_{2}\right)$ hold. On the other hand, H.J. Bremermann $[10]^{12)}$ proved the following important relation, whose proof is omitted here:

PROPOSITION 12. $\left(\mathrm{C}_{2}\right) \subset\left(\Gamma_{1}\right)$ holds.

Therefore, summing up the Propositions 8, 9, 10, 11 and 12, we obtain :

THEOREM 1. The ten classes of domains $\left(\mathrm{C}_{0}\right),\left(\mathrm{C}_{1}\right),\left(\mathrm{C}_{1}^{*}\right),\left(\mathrm{C}_{2}\right),\left(\Gamma^{0}\right)$, $\left(\Gamma^{\prime}\right),\left(\Gamma^{\prime}\right),\left(\Gamma_{*}\right),\left(\Gamma_{*}^{\prime}\right)$ and $\left(\Gamma_{1}^{\prime}\right)$ are all identical with one another.

Corollary 1. A domain $D$ is P-convex if and only if it is the limit of an exhaustion of $\mathrm{P}$-convex domains. In reaiity, we can select an exhaustion belonging to the class $\left(\mathrm{C}_{1}^{\gamma}\right)$.

Corollary 2. An (unbounded) domain D is P.convex if and only if for every hypersphere $B, B \cap D$ is always $\mathrm{P}$-convex.

In fact, the necessity is trivial because a hypersphere is P-convex. Conversely, taking $B$ sufficiently large, the last condition implies that $-\log d(z)$ is p.s. h. at every point $z$ of $D$, which implies $D \in\left(I^{\prime}\right)$.

Also from $\left(C_{0}\right)=\left(I^{\prime}\right)$, we have (Lelong [23], p. 203):

THEOREM 2 (Continuation theorem). If two domain $D_{1}, D_{2}$ are P-convex and $D_{1} \cap D_{2} \neq \emptyset$, but putting $G_{i} \equiv D_{i}-D_{1} \cap D_{2}(i=1,2), \overline{G_{1}} \cap \overline{G_{2}}$ $=\emptyset$ in the finite space, then $D_{1} \cup D_{2}$ is also P-convex.

CoROllary. For two parallel hyperplanes $A, B$ in 5 (5) we denote

12) pp. 50-54. See also Lelong [23], pp. 198-199. 
by $\subseteq(A ; B)$ the one of two half-spaces separated by $A$ containing $B$. If $D \cap \mathfrak{S}(A ; B)$ and $D \cap \mathfrak{S}(B ; A)$ are $\mathrm{P}$-convex, the domain $D$ itself is P-convex.

Using this continuation theorem,

Proposition 13. We have $\left(\mathrm{C}_{0}\right)=\left(\mathrm{C}_{0}^{l}\right)$.

Also from $\left(\mathrm{C}_{0}\right)=\left(I_{1}{ }_{1}\right)$ we have the following two propositions :

Proposition 14. When $n \geq 3$, a domain $D$ is $\mathrm{P}$-convex if and only if for every analytic hyperplane of real dimension $2 n-2, P$ : $a_{1} z_{1}+\cdots+a_{n} z_{n}=c, \quad c$ being a constant, each component of $P \cap D$ is always $\mathrm{P}$-convex in $5^{n-1}$.

Proposition 15. When $n \geq 3$, a domain $D$ is P-convex if and only if for every analytic bi-plane of real dimension $4, Q: z_{j}=z_{j}^{0}+a u+b v$, each component of $Q \cap D$ is always P-convex in $\mathbb{S}^{2}$.

Concerning the "radius of regularity" we have

PROPOSITION 16. A Hartogs domain $\mathscr{D} \equiv \equiv\{z \in D,|w|<\Phi(z)\}$ in the space $\mathbb{S}^{n+1}\left[z_{1}, \cdots, z_{n} ; w\right]$ is $\mathrm{P}$-convex if and only if $D$ is P.convex in the space $\left[\mathbb{S}^{n}(z)\right.$ and $-\log \Phi(z)$ is p. s. h. in $D$.

In fact, if $D$ is P.convex, taking a $=\left(a_{1}, \cdots, a_{n}, 0\right)$, we have $D \in\left(I_{1}\right)$, and taking $\mathrm{a}=(0, \cdots, 0,1),-\log \Phi(z)$ is p. s. h. in $D$. Conversely, putting

$$
\mathfrak{D}_{m} \equiv\{(z ; w)|-\log d(z)<m, \log | w \mid<\log \Phi(z)-1 / m\},
$$

we have $\mathscr{D}=\cup \mathscr{D}_{m}$ and then the condition implies that $\mathscr{D} \in\left(\mathrm{C}_{1}\right)$.

COROLlaRY. Let $Q(z)$ be the radius of regularity of a holomorphic function $f\left(z_{1}, \cdots, z_{n} ; w\right)$. Then $-\log Q(z)$ is p.s. h.

This last statement was first given by Hartogs [16], $\S 9$ for $n=1^{13}$, and has played a role of fundamental principle in the Rothstein's theory (W. Kothstein [31], [32]).

\section{§6. Levi pseudo-convex domains.}

Definition 14. A domain $D$ is said to belong to the class $\left(\mathrm{C}_{3}^{\alpha}\right)$, if for every boundary point $p$ of $D$, there exists a sphere $B$ with center at $p$ and a function $\Phi_{p}$ satisfying the condition of Levi-Krzoska in $B$ such that $D \cap B$ coincides with the set $B \cap\left\{\Phi_{p}<0\right\}$.

DEFINITION 15. A domain $D$ is called Levi strongly pseudo-

13) See also Bochner-Martin [8], p. 143. Lelong gives a generalization of this corollary using the continuation theorem. (Lelong [23], p. 206). 
conve $^{14)}$ if for every boundary point $p$ of $D$, there exists an analytic variety $\mathfrak{S}$ passing $p$ which lies entirely outside $D$ in some neighborhood around $p$, except the point $p$ itself. We denote the family of all Levi strongly pseudo convex domains by $\left(\mathrm{C}_{4}^{\alpha}\right)$, and especially when the variety 93 can be chosen as a quadratic variety, we write $D \in\left(\mathrm{C}_{4}^{\beta}\right)$. We denote by $\left(\mathrm{C}_{3}\right),\left(\mathrm{C}_{4}\right)$ or $\left(\mathrm{C}_{4}^{*}\right)$ the family of domains which are the limit of an exhaustion of domains belonging to $\left(\mathrm{C}_{3}^{\alpha}\right),\left(\mathrm{C}_{4}^{\alpha}\right)$ or $\left(\mathrm{C}_{4}^{\beta}\right)$, respectively.

From their definitions, it is evident that $\left(\mathrm{C}_{1}^{\gamma}\right) \subset\left(\mathrm{C}_{3}^{\alpha}\right)$ and $\left(\mathrm{C}_{4}^{\beta}\right) \subset\left(\mathrm{C}_{4}^{\alpha}\right)$. Also by Corollary to Proposition 6, we have $\left(C_{3}^{\alpha}\right) \subset\left(C_{4}^{\beta}\right)$. Therefore the inclusions $\left(\mathrm{C}_{0}\right)=\left(\mathrm{C}_{1}^{*}\right) \subset\left(\mathrm{C}_{3}\right) \subset\left(\mathrm{C}_{4}^{*}\right) \subset\left(\mathrm{C}_{4}\right)$ hold.

Proposition 17. A domain $D$ belonging to $\left(\mathrm{C}_{4}^{\beta}\right)$ is Cartan pseudoconvex.

In fact, for every boundary point $p$ of $D$, there exists a sphere $B$ with center at $p$, such that for every boundary point $q$ of $D$ in $B$, there exists a quadratic function $f_{q}(z)$ vanishing at $q$ and having no zero in $D \cap B$. For a boundary point $q$ of $B$ in $D$, there exists a tangential analytic hyperplane $f_{q}(z) \equiv a_{1} z_{1}+\cdots+a_{n} z_{n}-c=0$ which does not meet with $D \cap B$. Hence every boundary point $q$ of $D \cap B$ possesses a function $1 / f_{q}(z)$ holomorphic in $D \cap B$ with a singularity at $q$. Therefore a well-known criterion of Thullen $([36]$, "Satz 5 ") tells that $D \cap B$ is a domain of regularity.

More generally, E. E. Levi [25] and J. Krzoska [18] have proved that a Levi strongly pseudo-convex domain is of class $\left(\mathrm{C}_{4}^{\beta}\right)$ and so is Cartan pseudo-convex. Hence using this, we have :

THEOREM 3. $\left(\mathrm{C}_{4}^{\beta}\right)=\left(\mathrm{C}_{4}^{\alpha}\right) \subset\left(\mathrm{C}_{0}\right)=\left(\mathrm{C}_{3}\right)=\left(\mathrm{C}_{4}\right)=\left(\mathrm{C}_{4}^{*}\right)$ holds.

This theorem is also proved by Lelong [23] showing the relation $\left(\mathrm{C}_{4}^{\alpha}\right) \subset\left(\Gamma_{1}\right)$ directly, without using the above results due to Levi and Krzoska.

\section{Relations among the conjectures.}

\section{$\S 7$. Conjectures on the pluri-subharmonic functions.}

Concerning the Hartogs functions ( $\$ 3)$, we shall consider:

CONJECTURE 1 (Conjecture of Bochner-Martin). The family of

14) A domain of this kind has been called by several names. In Behnke-Thullen [6], it is called "total pseudokonvex", in Bochner [7], "strongly-convex", and in BehnkeStein [4], [5], "lokal analytisch konvex". 
p.s.h. functions will coincide with the family of modified Hartogs functions.

CONJECTURe 1 bis. The family of q.p.s. h. functions will coincide with the family of Hartogs functions.

CONJECTURe 2. A p.s.h. function $V(z)$ in $D$ will be represented in the form

$$
V(z)=\lim _{m \rightarrow \infty} \sup ^{*}\left(a_{m} \log \left|f_{m}(z)\right|\right)
$$

where all $a_{m}$ are non-negative constants and all $f_{m}(z)$ are one-valued holomorphic functions in D. (Lelong [22], Bremermann [10]).

CONJECTURE 3. If a domain $B$ is an analytic completion of $D$, i. e., if every function holomorphic in $D$ is automatically holomorphic in $B$, then every pluri-subharmonic function in $D$ will have $a$ p.s. $h .-$ continuation (cf. Definition 2) in B. (Lelong [22]).

THEOREM 4. The conjecture 2 implies the conjecture 1 and the conjecture 1 implies the conjecture 3.

PROoF. The first part is evident from Corollary to Proposition 7. Next, it is evident from their definitions that every modified Hartogs function in $D$ has the continuation as a modified Hartogs function in an arbitrary analytic completion of $D$. Therefore the second part is proved.

Lelong ([19]) has proved that the conjecture 2 is true when $n=1$, but unfortunately his method of demonstration of this fact depends upon the Riesz decomposition of a subharmonic function into the sum of a harmonic function (i. e., the real part of an analytic function) and a logarithmic potential. ${ }^{15)}$ Hence it is not applicable directly to the case of $n \geqq 2$. He also proved the following ${ }^{15)}$ :

Proposition 18. When a p.s.h. function $V(z)$ depends only on $r_{j} \equiv\left|z_{j}\right|(j=1, \cdots, n)$ in a Reinhardt domain, it is a modified Hartogs function. Moreover, $V(z)$ has the form

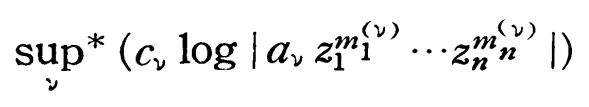

where $c_{\nu}$ are positive constants, and $m_{j}^{(\nu)}$ are positive integers.

A similar result holds for a p.s.h. function depending only on $x_{\boldsymbol{g}} \equiv \mathfrak{R} z_{j}$ in a tube domain ${ }^{16)}$.

15) See Radó [30], Chap. VI.

16) Lelong [20], § VIII, and [22]. For the case of tube, see [22]. 
Proposition 19. The conjectures 1 and 1 bis are equivalent.

PROOF. Since the operation of upper-regularization $\varphi \rightarrow \phi^{*}$ is commutative with the operation (H1) and (H2), the conjecture 1 bis implies the conjecture 1 because of Proposition 7. Conversely, as Brelot [9] has remarked, a q. p. s. h. function $U(z)$ is represented in the form $\sup \{V(z)\}$, $V(z)$ being p.s. h. functions majorized by $U(z)$ in $D$. Therefore the conjecture 1 implies the conjecture 1 bis.

Next we consider the domain of regularity.

Conjecture 4a (Conjecture of Lelong). A P-convex domain will be a domain of regularity, i.e., an Oka pseudo-convex domain will be a domain of regularity.

THEOREM 5. Conjecture 3 implies the conjecture 4a.

Proof. If a domain $D$ is $\mathrm{P}$-convex but is not a domain of regularity, $D$ must have an analytic completion $B$ strictly greater than $D$ itself. Then every p.s.h. function in $D$ will be continued in $B$ by the conjecture 3 . On the other hand, since $D \in\left(\mathrm{C}_{0}\right)=\left(\boldsymbol{I}_{0}\right)$, there exists a function $V(z)$ p.s. h. in $D$ which tends to $+\infty$ on every boundary point of $D$, and then such a function can be continued no longer, contradicting the above statement.

\section{$\S 8$. The problem of Levi.}

Concerning this, we state four more conjectures.

CONJECTURE 4b (Problem of Levi). Every Levi strongly pseudo. convex domain will be a domain of regularity.

CONJECTURE 4c (Conjecture of Oka). The continuation theorem of Oka which has been proved for $n=2$, holds still for any number of variables. ${ }^{17)}$ More precisely, if, for two parallel hyperplanes $A$ and $B$, the domain $D \cap \subseteq(A ; B)$ and $D \cap \subseteq(B ; A)$ are both domains of regularity, then the domain $D$ itself will be a domain of regularity.

CONJECTURE $4 \mathrm{~d}$. When $n \geq 3$, a domain $D$ is a domain of regularity if and only if for every analytic hyperplane of real dimension

17) For $n=2$, see Oka [26]. The author once said that this conjecture is true (Hitotumatu [17]), but the proof was incomplete. After W. Saxer [33], H. J. Bremermann obtained the same result. (His results was published quite recently in Math. Ann.). Recently Oka [27] applied the theory of ideals of analytic functions to this problem. (For the theory of ideals see for example, H. Cartan [14]). 
$2 n-2, \quad P: a_{1} z_{1}+\cdots+a_{n} z_{n}=c$, every component of $P \cap D$ is always $a$ domain of regularity in the space $\overleftarrow{5}^{n-1}$.

CONJECTURE $4 \mathrm{e}$. When $n \geq 3, a$ domain $D$ is a domain of regularity if and only if for every analytic bi-plane of real dimension 4, $Q: z_{j}=z_{j}^{0}+a u+b v$, every component of $Q \cap D$ is always a domain of regularity in the space $\mathfrak{5}^{2}$.

In the last two conjectures, the necessity is trivial, and the conjectures concern only on the sufficiency part.

THEOREM 6. The five conjectures $4 \mathrm{a}, 4 \mathrm{~b}, 4 \mathrm{c}, 4 \mathrm{~d}$ and $4 \mathrm{e}$ are equivalent with one another.

Proof. 4a implies the other four, because of Theorem 3, Corollary to Theorem 2, Proposition 14 and Proposition 15 respectively.

4b implies 4a. By Theorem 3, a P-convex domain is the limit of an exhaustion consisting of Levi strongly pseudo-convex domains $D_{m}$, and so $D_{m}$ is the limit of an exhaustion of domains of regularity provided that the conjecture $4 \mathrm{~b}$ holds. Then using a well-known theorem of Behnke-Stein [3], the domain $D$ itself is a domain of regularity.

$4 \mathrm{c}$ implies $4 \mathrm{a}$. If conjecture $4 \mathrm{c}$ is true, every Cartan pseudo-convex domain is a domain of regularity. Now a P.convex domain $D$ is the limit of an exhaustion of domains $D_{m}$ belonging to $\left(C_{4}^{\beta}\right)$, and $D_{m}$ are Cartan pseudo-convex by Proposition 17. Therefore, using again the theorem of Behnke-Stein, $D$ is a domain of regularity.

$4 \mathrm{e}$ implies $4 \mathrm{a}$. Since Oka [26] has proved that the conjecture 4c is true for $n=2$, the conjecture 4 a is valid for $n=2$. Hence if $D$ is P-convex in $\mathfrak{S}^{n}$, for every analytic bi-plane $Q, D \cap Q$ being P-convex in $\mathbb{S}^{2}$, all $D \cap Q$ are domains of regularity, which implies that the original domain $D$ itself is a domain of regularity provided that the conjecture $4 \mathrm{e}$ is true.

$4 \mathrm{~d}$ implies $4 \mathrm{e}$. For $n=3,4 \mathrm{~d}$ is the same thing to $4 \mathrm{e}$. Therefore we use the induction on the number of variables $n$, and assume that 4 e holds for $n-1$. Let $Q \cap D$ be a domain of regularity for any analytic bi-planes $Q$. For any analytic hyperplane $P$, we can select $Q$ contained in $P$, and then the domain $D \cap Q=(D \cap P) \cap Q$ is always a domain of regularity in $\mathbb{S}^{2}$.' Hence by the assumption of induction, $D \cap P$ is a domain of regularity in $\mathbb{C}^{n-1}$ which implies that $D$ is also a domain of regularily in ${ }^{5} n$ provided that the conjecture $4 \mathrm{~d}$ holds.

Thus our theorem is completely proved. 
On some conjectures concerning pseudo-convex domains.

We shall write conjecture 4 for any one of the conjectures $4 \mathrm{a}-4 \mathrm{e}$.

\section{§9. Problems on the Hartogs domains.}

CONJeCture 5. A Hartogs domain $\mathfrak{D}=\{z \in D,|w|<R(z)\}$ in the space ${ }^{n+1}[z ; w]$ is a domain of regularity if and only if $D$ is a domain of regularity in $\left[5^{n}[\mathrm{z}]\right.$ and $-\log R(z)$ is p.s.h. in $D$.

The necessity of this statement is so easily proved, that the significance of the conjecture is only for its sufficiency part. This conjecture is true for $n=1$. (Bremermann [10]).

More generally, we put the following but somewhat artifical one:

CONJECTURe 6. For a P-convex domain $D$ there exists $a$ p.s. $h$. function $V(z)$ in $D$ such that the Hartogs domain $D \equiv\{z \in D,|w|<$ $\exp (-V(z))\}$ is a domain of regularity in the space $\mathbb{C}^{n+1}[z ; w]$.

THEOREM 7. The conjecture 6 is equivalent to the conjecture 4 and implies the conjecture 5 .

Proof. By Proposition 16, it is easy to see that the conjecture 4a for $(n+1)$ variables implies the conjecture 5 .

Also, if the conjecture $4 \mathrm{a}$ is true for $n$ and $n+1$ variables, the conjecture 6 holds for any p.s. h. function $V(z)$ in $D$.

Conversely, when the conjecture 6 is true, a P-convex domain $D$ is a section of a domain of regularity $D$ in the space $\mathfrak{C}^{n+1}$ by a hyperplane $w=0$, and $D$ is then a domain of regularity.

THEOREM 8. The conjecture 5 is equivalent to the conjecture 2 restricted only on the domains of regularity.

Proof. By one of the important results of Cartan-Thullen [15], a Hartogs domain of regularity is a convergence region of a suitably chosen Hartogs series $\sum_{m=0}^{\infty} f_{m}(z) w^{m}$. When $D$ is a domain of regularity and $V(z)$ is p.s. h. in' $D$,

$$
\mathcal{D} \equiv\{(z ; w)|z \in D,| w \mid<\exp (-V(z))\}
$$

being the convergence region of $\sum_{m=0}^{\infty} f_{m}(z) w^{m}$ provided that the conjecture 5 holds. Therefore we have by the formula of convergence radius, ${ }^{18)}$

18) Hartogs [16]. See also Rothstein [31]. 


$$
V(z)=\limsup _{m \rightarrow \infty} *\left(\frac{1}{m} \log \left|f_{m}(z)\right|\right) .
$$

Conversely, let $D$ be a domain of regularity and let a function $V(z)$ p. s. h. in $D$ have the form

$$
V(z)=\lim \sup ^{*}\left(a_{m} \log \left|f_{m}(z)\right|\right), \quad\left(a_{m}>0\right) .
$$

First we show that a domain $\left(\mathfrak{B} \equiv\left\{z \in G,|w|<\exp \left(-\frac{r}{s} \log |\phi(z)|\right)\right\}\right.$ is a domain of regularity, where $G$ is a domain of regularity in $\mathbb{C}^{n}, \phi(z)$ is holomorphic in $G$ and $r, s$ are positive integers. In fact, for a boundary point $p$ of $\mathbb{B}$ whose projection on $G$ lying on the boundary of $G$, we give a function $\chi(z)$ having the boundary of $G$ as its natural boundary. For a boundary point $p=\left(z_{0} ; w_{0}\right)$ of $(B)$ with $\left|w_{0}\right| s\left|\varphi\left(z_{0}\right)\right|^{r}=1$, we give a function

$$
1 /\left[w^{s} \cdot \phi(z)^{r}-e^{i \theta}\right]
$$

where $e^{i \theta} \equiv w_{0}^{s} \cdot \varphi\left(z_{0}\right)^{r}$. Therefore, by the criterion of Thullen ([36]), (G) is a domain of regularity. Next we show that if $\phi(z)$ is holomorphic and bounded in $G$, the domain

$$
\widetilde{\mathfrak{S}} \equiv\{z \in G,|w|<\exp (-a \log |\varphi(z)|)\}, \quad(a>0)
$$

is also a domain of regularity. For, if $|\varphi|<M$ in $G$, we have

$$
\widetilde{\mathfrak{S}}=\bigcap_{\boldsymbol{b}} \mathscr{G}_{b} ; \mathfrak{G}_{b} \equiv\left\{z \in G, M^{a-b}|w|<\exp (-b \log |\varphi(z)|)\right\},
$$

where $b$ runs over all the positive rational numbers greater than $a$, and since $\mathscr{B}_{b}$ are domain of regularity, so is their intersection $\widetilde{\mathfrak{S}}$.

Now a domain of regularity $D$ is the limit of an exhaustion of domains of regularity $D_{k}$ (in reality, an exhaustion of polyhedral domains) and since $V(z)=\lim _{m \rightarrow \infty} \sup ^{*}\left(a_{m} \log \left|f_{m}(z)\right|\right)$ we have $\exp (-V(z))$ $\left.=\lim _{l \rightarrow \infty}\left[\inf _{m \geq l} \exp \left(-a_{m} \log \left|f_{m}(z)\right|\right)\right]_{*} \cdot{ }^{19}\right) \quad$ We have already proved that the domains

$$
\mathfrak{D}_{k, m} \equiv\left\{z \in D_{k},|w|<\exp \left(-a_{m} \log \left|f_{m}(z)\right|\right)\right\}
$$

are all domains of regularity, since $f(z)$ is holomorphic and bounded in $D_{k}$. Therefore the domain

19) $\Psi_{*}^{*}(z)$ means the "lower regularization" $\lim _{\zeta \rightarrow z} \inf \Psi(\zeta)$. 


$$
\widetilde{\mathfrak{G}}_{k, l} \equiv\left\{z \in D_{k},|w|<\left[\inf _{m \geq l} \exp \left(-a_{m} \log \left|f_{m}(z)\right|\right]_{*}\right\}=\underset{m \geq l}{\bigcap} \mathfrak{D}_{k, m}\right.
$$

being also a domain of regularity, and choosing a suitable subsequence $\mathfrak{D}_{j} \equiv \widetilde{\mathfrak{D}}_{k_{j}, l_{j}}$ from $\left\{\widetilde{\mathfrak{D}}_{k, l}\right\}$, $\mathfrak{D}$ is the limit of an exhaustion $\mathfrak{D}_{j}$ consisting of domains of regularity. Hence $\mathfrak{D}$ itself is a domain of regularity, which proves our assertion.

COROLLARY. If the conjecture 3 is true, the conjecture 2 holds for the domains with univalent envelope of regularity. ${ }^{20)}$

Proof. Since the conjecture 3 implies 5 by Theorems 6 and 7, a function $V(z)$ p.s.h. in a domain of regularity has the form $\limsup _{m \rightarrow \infty}\left(a_{m} \log \left|f_{m}\right|\right)$. If the envelope of regularity $B$ of $D$ is univalent, a p.s.h. function in $D$ is continued into $B$ provided that the conjecture 3 is true, and so has the form as in the conjecture 2. Contracting it to the original domain $D$, we have our assertion.

It seems to us that this Corollary is true for an arbitrary domain, i. e., the above additional assumption for the domain to have univalent envelope of regularity will not be necessary.

THEOREM 9. For a Reinhardt domain, the conjecture 4a is true. ${ }^{21)}$

Proof. If a Reinhardt domain $\mathfrak{D}$ in the space $\mathbb{C}^{n+1}[z ; w]$ is $\mathrm{P}$ convex, D has the form as a Hartogs domain $\{z \in D,|w|<R(z)\}$ where $D$ is a Reinhardt P-convex domain in $5^{n}[z]$ and $R(z)$ depends only on $\left|z_{j}\right|$. A p.s. h. function $-\log R(z)$ has then the form

$$
\sup _{\nu} *\left(a_{\nu} \log \left|c_{\nu} z_{1}^{m_{1}^{(\nu)}} \cdots z_{n}^{m_{n}^{(\nu)}}\right|\right)
$$

by Proposition 18. Now for $n=2$, the conjecture $4 \mathrm{a}$ is true by the results of Oka [26]. Then using the induction of $n$, we may assume that our statement has been proved for $\left[5^{n}\right.$, and so $D$ is a domain of regularity. Then an analogous process in the demonstration of Theorem 8 shows that the domain

20) The envelope of regularity means the maximum analytic completion (Regularitätshülle).

21) This was first given by Almer [1]. Thullen [37] proved that, for a Reinhardt domain, the three properties "pseudo-convex", "domain of regularity" and "domain of meromorphy" coincide with each other. 


$$
\mathfrak{D}=\{z \in D,|w|<R(z) \equiv \exp [-(-\log R(z))]\}
$$

is a domain of regularity which proves our assertion for $(n+1)$ variables.

\author{
Department of Mathematics, \\ Saint Paul's University, Tokyo.
}

\title{
References
}

[1] Almer, B., Sur quelques problèmes de la théorie des fonctions analytiques de deux variables complexes, Arkiv för Mat. Astron. och Fys., 17, No. 7 (1922), 1-70.

[2] Behnke, H. and Sommer, F., Über die Voraussetzungen des Kontinuitätssatzes, Math. Ann, 121 (1950), 356-378.

[3] Behnke, H. and Stein, K., Konvergente Folge von Regularitätsbereichen und die Meromorphiekonvexität, Math. Ann., 116 (1938), 204-216.

[4] Behnke, H. and Stein, K., Die Konvexität in der Funktionentheorie mehrerer komplexer Veränderlichen, Mitteilungen der Math. Ges. in Hamburg, 8 (1940), 34-81.

[5] Behnke, H. and Stein, K., Die Singularitäten der analytischen Funktionen mehrerer Veränderlichen, Nieuw Archief voor Wiskunde, 23 (1951), 227-242.

[6] Behnke, H. and Thullen, P., Theorie der Funktionen mehrerer komplexer Veränderlichen, Ergebn. d. Math., 3, Berlin, 1934.

[7] Bochner, S., Functions of several complex variables, Princeton Lecture, Mimeographed Note, 1936.

[8] Bochner, S. and Martin, W. T., Several complex variables, Princeton, 1948.

[9] Brelot, M., Sur le rôle du point à l'infini dans la théorie des fonctions harmoniques, Ann. F́cole Norm. Sup., (3) 61 (1941), 301-332.

[10] Bremermann, H. J., Die Charakterisierung von Regularitätsgebieten durch pseudokonvexe Funktionen, Dissertation Münster, Schriftenreihe des Math. Inst. Univ. Münster, 5 (1951), 1-92.

[11] Cartan, H., Sur les domaines d'existence des founctions de plusieurs variables complexes, Bull. Soc. Math. France, 59 (1931), 46-69.

[12] Cartan, H., Théorie générale du balayage en potentiel newtonien, Ann. Univ. Grenoble, 22 (1946), 221-280.

[13] Cartan, H., Problèmes globaux dans la théorie des fonctions analy tiques de plusieurs variables complexes, Proc. International Congress of Math. at Cambridge Mass. 1950, I (1952), 152-164.

[14] Cartan, H. et al., Séminaire H. Cartan, Paris, 1951/52.

[15] Cartan, H. and Thullen, P., Regularitäts- und Konvergenzbertiche, Math. Ann., 106 (1932), 617-647.

[16] Hartogs, F.. Zur Théorie der analytischen Funktionen mehrerer unabhängiger Veränderlichen, insbesondere über die Darstellung derselben durch Reihen, welche nach Potenzen einer Veränderlichen fortschreiten, Math. Ann., E2 (1906), 1-88.

[17] Hitotumatu, S., On integral formulas of analytic functions of several complex 
variables and some related problems, Kōdai Math. Sem. Reports, 1, No. 5/6 (1949), 11-15.

[18] Krzoska, J., Über die natürlichen Grenzen analytischer Funktionen mehrerer Veränderlicher, Dissertation Greifswald, 1933, 1-21.

[19] Lelong, P., Sur quelques problèmes de la théorie des fonctions de deux variables complexes, Ann. École Norm. Sup., (3) 58 (1941), 83-177.

[20] Lelong, P., Les fonctions plurisousharmoniques, Ann. École Norm. Sup., (3) 62 (1945), 301-338.

[21] Lelong, P., Propriétés métriques des variétés analytiques complexes définies par une équation, Ann. École Norm. Sup., (3) 67 (1950), 393-419.

[22] Lelong, P., La convexité et les fonctions analy tiques de plusieurs variables complexes, Jour. de Math. pure et appl., (9) 31 (1952), 191-219.

[23] Lelong, P., Domaines convexes par rapport aux fonctions plurisousharmoniques, Journ. d'Analyse Math., 2 (1952), 178-208.

[24] Levi, E E., Studii sui punti singolari essenziali delle funzioni analitiche di due o più variabili complesse, Annali di Mat. pura ed appl., (3) 17 (1910), 61-87.

[25] Levi, E. E., Sulle ipersuperficie dello spazio a 4 dimensioni che possono essere frontiera del campo di esistenza di una funzione analitica di due variabili complesse, Annali di Mat. pura ed appl., (3) 18 (1911), 69-79.

[26] Oka, K., Sur les fonctions analytiques de plusieurs variables VI, Domaines pseudoconvexes, Tôhoku Math. Journ., 49 (1942), 15-52.

[27] Oka, K.,-VIII, Lemme fondamentale, Journ. Math. Soc. Japan, 3 (1951), 204-212; 259-279.

[28] Oka, K.,-IX, Domaines finis sans point critique intérieur, Jap. Journ. of Math., 23 (1953), 97-155.

[29] Osgood, W. F., Lehrbuch der Funktionentheorie, II, 1, Leipzig, 2nd ed., 1929.

[30] Radó, T., Subharmonic functions, Ergebn. d. Math., 5, Berlin, 1937.

[31] Rothstein, W., Ein neuer Beweis des Hartogsschen Hauptsatzes und seine Ausdehnung auf meromorphe Funktionen, Math. Zeits. 53 (1950), 89-97.

[32] Rothstein, W., Zur Theorie der Singularitäten analy tischer Funktionen und Flächen, Math. Ann., 126 (1953), 221-238.

[33] Saxer, W., Sur les domaines de normalité des fonctions méromorphes de plusieurs variables, Journ. de Math. pure et appl., (9) 31 (1952), 49-53.

[34] Schwartz, L., Théorie des distributions, I, II, Actual. Sci. Ind., Paris, 1950/51.

[35] Thorin, G. O., Convexity theorem, Meddelanden Från Lunds Univ. Mat. Sem., 9 (1948), 1-58.

[36] Thullen, P., Die Regularitätshüllen, Math. Ann., 106 (1932), 64-76.

[37] Thullen, P., De la teoría de las funciones analíticas de varias variables complejas. Dominios de regularidad y dominios de meromorfía de Reinhardt, Revista Unión Mat. Argentina, 11 (1945), 33-46.

[38] Thullen, P., Problemas de la teoria de las funciones analiticas de varias variables complejas, Symposium Mat. Latino America, Montevideo, 1951, 107-119.

[39] Weil, A., L'intégrale de Cauchy et les fonctions de plusieurs variables, Math. Ann., 111 (1935), 178-182. 